\title{
Radiofrequency-assisted liver resection: short-term results in a single institution
}

\author{
Ihnat $\mathrm{P}^{1}$, Vavra $\mathrm{P}^{1,2}$, Rudinska $\mathrm{L}^{3}$, Ostruszka $\mathrm{P}^{1}$, Dostalik $\mathbf{J}^{1}$ \\ Department of Surgery, University Hospital Ostrava, Czech Republic. peterihnat@yahoo.com
}

\begin{abstract}
Objectives: The main goal of the study was to evaluate the effectiveness and safety of radiofrequency-assisted liver resection.

Background: Liver resection is the "gold standard" for patients with resectable liver tumors. In the past years, the role of radiofrequency in liver surgery has been expanded from simple tumor ablation to its use in the technique of radiofrequency-assisted liver resection.

Methods: Patients with primary or secondary liver tumors, who underwent radiofrequency-assisted liver resection have been included into the prospective study. The acquired data underwent statistical analysis and were compared with the published results of liver resections.

Results: Between January 1, 2007 and September 30, 2009, 53 patients underwent radiofrequency-assisted liver resection. Seventy-six tumours were resected with the mean diameter of $38 \pm 19 \mathrm{~mm}$. Mean peroperative blood loss was $170.8 \pm 285.4 \mathrm{~mL}$ and transfusions were needed postoperatively in $9.4 \%$ cases. The mean hospital stay was $10.6 \pm 7.2$ days. Postoperative complications were noted in $16.9 \%$ patients; postoperative mortality was $1.9 \%$. Conclusion: The radiofrequency-assisted liver resection represents a safe and effective way of hepatic parenchyma transaction and to hepatobiliary surgeon it offers a new way of effective transection of liver parenchyma (Tab. 2, Ref. 23). Full Text in PDF www.elis.sk.

Key words: radiofrequency, liver tumors, liver resection.
\end{abstract}

Primary and secondary liver tumors present a challenging problem in clinical oncology because they bring about very high morbidity and mortality. Hepatic resection is accepted as the best treatment for resectable liver tumors as it leads to a significantly improved survival. Unfortunately, as a result of the extent and localization of tumor, inadequate hepatic reserve or because of very high patient's co-morbidity. The resection of hepatic malignancies is possible in only $10-25 \%$ of patients at the time they are first seen $(1,2)$.

Patients with unresectable disease may be candidates for local ablative techniques, chemoembolization, systemic or local chemotherapy (2-4). Radiofrequency thermal ablation (RFA) has been increasingly used for unresectable hepatic tumors (3). Multiple trials have evaluated RFA for the treatment of unresectable primary and secondary liver tumors and proved that RFA can control hepatic malignancies with few associated complications (2-6).

Surgical treatment is the "gold standard" for patients with liver metastases or primary liver tumors. Intraoperative blood loss and the subsequent need for blood transfusion are considered significant risk factors for increased mortality and morbidity, poor postoperative outcome and shorter duration of the disease-free interval

${ }^{1}$ Department of Surgery, University Hospital Ostrava, Czech Republic, ${ }^{2} \mathrm{HPB}$ unit, Division of Surgery, Hammersmith Hospital, Imperial College London, UK, and ${ }^{3}$ Department of pathology, University Hospital Ostrava, Czech Republic

Address for correspondence: P. Ihnat, MD, Dept of Surgery, University Hospital Ostrava, 17. listopadu 1790, CZ-708 52 Ostrava, Czech Republic. Phone: +420.777 .571880$
(7-8). Over the years, various techniques have been developed to allow safer liver resection.

The role of radiofrequency (RF) energy in liver surgery has been expanded in the past years from simple tumor ablation to its use in the technique of radiofrequency-assisted liver resection. This new technique employs heat produced by a radiofrequency needle electrode to coagulate the liver tissue before cutting it, thus permitting liver resection with minimal blood loss $(7,9)$.

Thermal energy induced by radiofrequency generator is applied through special RF needle (Habib Sealer 4X) repeatedly along the intended resection line. This leads to creating an approximately 2-cm-wide zone of coagulative necrosis where liver parenchyma can be divided by scalpel with minimal blood loss $(7,9)$. Hemostasis is obtained only by radiofrequency energy; no additional devices (e.g. stitches, knots, clips or glue) are needed. The use of RF energy for liver resection was pioneered by Professor Nagy Habib, which is why it is also called Habib's resection.

RF-assisted liver resection is a simple method which can be used in open or minimally invasive surgery. RF-assisted technique of resection can be used for all types of liver resections from wedge resections to hemihepatectomies. It has been reported to be associated with minimal blood loss, low blood transfusion requirement and no need of intraoperative hepatic inflow occlusion techniques such as Pringle's maneuver, dissection and clamping of hepatic pedicle $(7,9-11)$. This innovative technique allows performing more minor hepatic resections and less major hepatectomies. and thus spares patient's normal hepatic parenchyma for future re-resections. 
In terms of disease recurrence there is a very important advantage of RF-assisted resection over standard liver resection techniques. The resection line is treated by thermal ablation, leaving a 1-cm-wide zone of coagulated tissue in situ. Consequently, lower disease recurrence in resection bed is anticipated, and in indicated cases it allows to drift the resection line closer to the tumor $(1,10,12)$.

\section{Methods}

During the study period (January 1, 2007 - September 30, 2009), we performed 53 radiofrequency-assisted hepatic resections in patients with primary and secondary liver tumors at the University Hospital, Ostrava, Czech Republic.

All patients underwent appropriate screening consisting of complete history, physical examination, laboratory testing including tumor markers, and computed tomography and/or magnetic resonance imaging to determine operability. The aim was to evaluate carefully the patients' general fitness for anesthesia and nutritive status, as well as their cardiovascular, hepatic and renal functions. Each patient was then discussed at the multidisciplinary meeting involving surgical oncologists, medical oncologists and radiologists, and based on latter consultations, indicated for liver resection.

The exclusion criteria for liver resection included extensive intrahepatic disease beyond the reach of multiple subsegmental resections to achieve total clearance and preservation of adequate liver parenchyma; presence of extrahepatic metastases detected prior to or during surgery; high operative risk due to poor performance status.

\section{The technique}

Under general anesthesia, diagnostic laparoscopy was performed. Patients with peritoneal disease were considered unsuitable for liver resection. Any suspicious lymph nodes or peritoneal nodules were submitted for frozen section histology. A thorough examination of the liver was performed with obligatory intraoperative ultrasonography (IOUS) to confirm the number, size, and location of the liver tumors (especially in relation to hepatic veins and portal sheaths).

As long as the liver lesions were suitable for laparoscopic resection, we have used the combination of hand-assisted laparoscopic technique (HALS) and RF-assisted resection technique using special laparoscopic bipolar RF probe (Laparoscopic Habib $4 X)$. This combined approach for liver resection was firstly used and published by our team (13). The principle of parenchyma transection is the same as in open approach described below.

In patients with liver tumors not suitable for laparoscopic approach, we continued the procedure with laparotomy. Right subcostal or J incision was done and the peritoneal cavity was examined for evidence of extrahepatic spread once more. Abdominal adhesions and the falciform ligament were divided; the liver lobe that contained the tumor was mobilized in a standard way to the extent necessary for the intended resection.

The detailed description of radiofrequency-assisted liver resection has been published previously by investigators who had first developed it $(7,9)$. In brief, a line of intended division of liver parenchyma was made on the liver capsule with diathermy $1 \mathrm{~cm}$ from the edge of the tumor in order to provide a roadmap for resection.
The RF probe (Habib Sealer 4X) was then repeatedly introduced into the liver parenchyma along the marked line to induce a zone of coagulative necrosis. Afterwards, the liver parenchyma was divided along the ablated zone by using the scalpel. There was no need to use any vascular inflow occlusion techniques while hemostasis was obtained purely by radiofrequency energy. At the same time no additional devices (e.g. stitches, knots, clips or glue) were needed. All patients had complete liver resection with at least $1-\mathrm{cm}$ surgical margin.

\section{Statistical analysis}

Patient data were collected prospectively, including demographic details, histopathological origin, number and localization of tumors, operative procedure, operating time, blood loss, blood transfusion requirement, morbidity, mortality and duration of hospital stay.

The acquired data underwent statistical analysis and were compared with the published results of liver resections. Results were expressed as either median (range) or mean \pm SD. Differences in means between subgroups were compared using the Student t-test. Comparisons between categorical variables were analyzed using the chi-squared test. Mann-Whitney U test was used to evaluate the differences between continuous variables. $p$ values $<0.05$ were considered statistically significant.

\section{Results}

In a prospective study, starting January 2007 and ending September 2009, a total of 53 radiofrequency-assisted resections were done. Clinical details of these patients are summarized in Table 1. There were 27 men (50.9\%) and 26 women (49.1\%) with mean age of $63.2 \pm 9.2$ years (range 39-82 years).

Histopathological origin of tumors in our study was in correlation with geographic distribution of primary and secondary liver tumors worldwide $(2,14,15)$. There have been only 2 patients (3.8\%) with primary liver tumors, while 41 patients $(77.4 \%)$ had secondary liver tumors - usually colorectal cancer metastasis (64.2\%). Lesions were benign in 3 patients $(5.7 \%)$.

Altogether 76 lesions were resected. Tumors were solitary in the majority of cases $(71.7 \%)$; less frequent were two $(15.1 \%)$ or more lesions in one patient. The average number of tumors per patient was $1.4 \pm 0.8$ (range $1-4$ ) with an overall mean size of 38 $\pm 19 \mathrm{~mm}$ (range $8-120 \mathrm{~mm}$ ).

As shown in Table 1, lesions were located in segments II VIII, most frequently in segments V and VI. Very commonly, the pathological lesions trenched on more than one segment; each patient had 1.9 segments stricken in average.

The types of performed liver resection are listed in Table 2. Surgical procedures ranged from simple wedge resection to right hepatectomy. We performed 35 non-anatomical liver resections (66\%) and 18 anatomical resections ( $34 \%$ ). The most frequent type of procedure was wedge resection which was done in 28 patients $(52.8 \%)$. Out of these 28 patients, in 7 cases, the wedge resection was done twice (wedge resections of 2 lesions in different liver segments in one patient) and in 1 case, a quadruple wedge resection was done. Major liver resection ( $\geq 3$ segments) was performed in 7 patients (13.2\%). 
Tab. 1. Patients clinical details.

\begin{tabular}{lc}
\hline Mean age in years & $63.2 \pm 9.2$ years (range, $39-82$ years) \\
\hline Sex $(\mathrm{M} / \mathrm{F})$ & $27 / 26$ \\
\hline Diagnosis & 2 \\
Hepatocellular carcinoma & 3 \\
Benign liver lesions & 34 \\
Colorectal cancer metastasis & 7 \\
Gallbladder carcinoma & 3 \\
Breast cancer metastasis & 2 \\
Melanoblastoma metastasis & 2 \\
Grawitz's tumor metastasis & $38 \pm 19 \mathrm{~mm}(\mathrm{range}, 8-120 \mathrm{~mm})$ \\
\hline Mean tumor size & $1.4 \pm 0.8($ range, $1-4)$ \\
\hline Mean number of lesions & 21 \\
\hline Location of tumor & 11 \\
Segment II/III & 24 \\
Segment IV & 19 \\
Segment V/VI & 1 \\
Segment VI/VII & \\
Segment VIII &
\end{tabular}

All the procedures were achieved according to the previously described technique of RF-assisted liver resection. None of the patients had vascular inflow occlusion (Pringle's maneuver). All the resection margins were free of tumor.

As shown in Table 2, out of 53 patients in our study group, 42 patients $(79.2 \%)$ were operated by means of laparotomy (after initial diagnostic laparoscopy). The liver resection was completed in hand-assisted laparoscopic fashion for 11 patients (20.8\%).

The mean operative time was $150.5 \pm 52.9$ min (range 50 $350 \mathrm{~min}$ ). The mean time necessary for transaction of the liver parenchyma was $56 \pm 23.1 \mathrm{~min}$ (range $32-105 \mathrm{~min}$ ).

The mean intraoperative blood loss was $170.8 \pm 285.4 \mathrm{~mL}$ (range $17-1450 \mathrm{~mL}$ ). A total of 5 patients $(9.4 \%$ ) received blood transfusion in intraoperative and postoperative periods, with a mean of 2.6 units (range $1-4$ ) of red blood cells per patient (in the subgroup of patients receiving blood transfusions).

During the first postoperative day, the secretion from abdominal drain (positioned in the liver resection bed) was $50.7 \pm 78.9$ $\mathrm{mL}$ (range $0-550 \mathrm{~mL}$ ). The drain was extracted after $5.1 \pm 2.5$ days (range $1-13$ days).

Patients after RF-assisted liver resection were all admitted to intensive care unit (according to our hospital policy) for postoperative monitoring and stayed there for $3.4 \pm 1.7$ days (range $1-9$ days). The mean hospital stay in our study group was $10.6 \pm 7.2$ days (range $3-44$ days).

Complications in the postoperative period were noted in 9 patients $(16.9 \%)$. The most frequent complication was wound infection observed in 3 patients $(5.7 \%)$. The formation of subhepatic abscess (in the resection bed) was seen in 2 patients $(3.8 \%$ ) and effectively managed by drainage under $\mathrm{CT}$ control, no reoperation was necessary. Pleural effusion on the right side developed also in two patients $(3.8 \%)$ and was managed by conservative treatment in both cases. One patient (after right hemihepatectomy) developed clinical and laboratory signs of temporary hepatic failure in the postoperative period and recovered after 6 days.

Within 30 days after the procedure, we have registered one death resulting in perioperative mortality rate of $1.9 \%$ in our study
Tab. 2. Type and results of resection.

\begin{tabular}{lc}
\hline Type of resection (n) & \\
Wedge resection & $28(52.8 \%)$ \\
Segmentectomy & $6(11.3 \%)$ \\
Bisegmentectomy & $6(11.3 \%)$ \\
Gallbladder bed resection & $6(11.3 \%)$ \\
Major hepatectomy ( $\geq 3$ segments) & $7(13.2 \%)$ \\
\hline Type of surgical access & $42(79.2 \%)$ \\
Laparotomy & $11(20.8 \%)$ \\
Hand - assisted laparoscopic surgery & $170.8 \pm 285.4 \mathrm{~mL}$ \\
\hline Mean blood loss (mL) & (range, $17-1450 \mathrm{~mL})$ \\
\hline Mean operative time (min) & $150.5 \pm 52.9 \mathrm{~min}$ \\
& (range, $50-350 \mathrm{~min})$ \\
\hline Mean resection time (min) & $56 \pm 23.1 \mathrm{~min}$ \\
\hline Mean length of hospital stay (days) & (range, $32-105 \mathrm{~min})$ \\
\hline
\end{tabular}

group. A 72-year-old patient after RF-assisted bisegmentectomy developed respiratory insufficiency and failure. It was followed by multiple organ failure (MODS) and death after 6 days.

\section{Discussion}

Perioperative morbidity and mortality rates after liver resection are very significantly influenced by intraoperative blood loss and requirement of blood transfusions in the perioperative period $(2,7-10)$. That is why various methods have been developed to minimize the intraoperative blood loss (vascular occlusion techniques and modern techniques of liver parenchyma transection such as water-jet dissector or CUSA).

While vascular occlusion techniques are effective in reducing the intraoperative blood loss, in patients with pre-existing chronic liver disease, they may increase morbidity and mortality $(1,2,16)$. Among modern techniques of liver parenchyma transection, radiofrequency-assisted resection has been increasingly used in the past years. Various authors have reported excellent effects of RFassisted resection technique on reducing the intraoperative blood loss $(7,9-12,17)$.

The mean intraoperative blood loss in our study group was 170.8 $\mathrm{mL}$ while transfusion was needed in $9.4 \%$ of patients. Values of intraoperative blood loss published in literature for liver resection by other techniques vary between $250 \mathrm{~mL}$ and $1700 \mathrm{~mL}$ with transfusion rates from $6.9 \%$ to $62.3 \%[(2,18-23)$. Statistical testing confirmed our results to be significantly better than most of previously published studies of patients after liver resection by other techniques.

The most extensive group of patients after RF-assisted liver resection (236 patients) was published by the team of professor Habib (7) with mean blood loss of $157 \mathrm{~mL}$ and blood transfusion given to $4 \%$ of patients. Our results are comparable with those of Professor Habib. There is no statistically significant difference in blood loss; the transfusion rate is slightly worse in our study group.

RF-assisted technique of resection allowed us to minimize the number of major hepatectomies. Major hepatectomy was performed only in $13.2 \%$ of patients, which is similar to Habib's rate of major hepatectomies (17\%). 
After liver resection, all our patients were observed postoperatively at the intensive care unit, which is in stark contrast to only $2.1 \%$ of patients admitted to intensive care unit in Habib's study. The difference is caused by varied health care habits developed in Czech Republic and United Kingdom. It is a common practice in our hospital to observe this kind of patients postoperatively at ICU. Mean hospital stay in our study group was 10.6 days. When compared with the hospital stay in published series of patients after liver resection (by RF-assisted or other techniques) ranging from 9 to 17 days $(2,18-23)$, it is statistically the same or better result.

The overall postoperative complication rate was $16.9 \%$ in this series, which is consistent with or even better than in most reported large series ranging from 16 to $45 \%(2,18-23)$. The complications included wound infection, subhepatic abscess, pleural effusion, respiratory and temporary hepatic failure. The number and the spectrum of complications were similar to those of patients after RF-assisted liver resection in professor Habib's study group.

Published series of patients after liver resection report mortality rates (within 30 days after operation) to be lower than $3 \%$. The mortality rate of $1.9 \%$ in our study group is comparable to those in published results.

\section{Conclusion}

In conclusion, it can be said that utilization of radiofrequency energy in performing bloodless liver resection seems to be very promising. Short-term results of our study (in accordance with other published studies of patients after RF-assisted liver resection) confirmed this technique to be very effective and simple. It is used mainly for non-anatomical wedge resections with very low blood loss, low need of blood transfusions (in less than $10 \%$ of patients), low postoperative morbidity (less than $25 \%$ ) and mortality (less than $3 \%$ ), pleural effusions and perihepatic collections being the most frequent complications managed conservatively in the majority of cases. For its excellent hemostatic effect it is an ideal tool also for laparoscopic liver resections.

Although the current study was not designed to compare RFassisted liver resection with other techniques, it seems to be better in terms of intraoperative blood loss, transfusion requirement, postoperative morbidity and mortality when compared with recently published large series on liver resection.

Currently, no comparative prospective studies exist in literature but the number of surgical groups committed to this new field of liver surgery is a proof that RF-assisted resection is about to become an exciting and challenging operation.

\section{References}

1. Skalický T, Třeška V, Šnajdauf J. Chirurgie jater. Praha: Maxdorf, 2004: $25-138$.

2. Blumgart LH et al. Surgery of the liver, biliary tract, and pancreas. 4th ed; Philadelphia; Saunders, 2007: 1269-1416.

3. Lencioni R, Crocetti L, Cioni D, Della Pina C, Bartolozzi C. Percutaneous radiofrequency ablation of hepatic colorectal metastases : technique, indications, results, and new promises. Invest Radiol 2004; 39: 689-697.
4. Strasberg SM, Linehan D. Radiofrequency ablation of liver tumors. Curr Prob Surg 2003; 40: 451-498.

5. Bowles BJ, Machi J, Limm WMLet al. Safety and efficacy of radiofrequency thermal ablation in advanced liver tumors. Arch Surg 2001; 136: 864-869.

6. Abdalla EK, Vauthey JN, Ellis LM et al. Recurrence and outcomes following hepatic resection, radiofrequency ablation, and combined resection/ablation for colorectal liver metastases. Ann Surg 2004; 239: 818-827.

7. Ayav BP, Habib NA, Pellicci R, Tierris J, Milicevic M, Jiao LR. Impact of radiofrequency assisted hepatectomy for reduction of transfusion requirements. AM J Surg 2007; 193: 143-148.

8. Jarnagin WR, Gonen M, Fong $Y$ at el. Improvement in perioperative outcome after hepatic resections: Analysis of 1803 consecutive cases over the past decade. Ann Surg 2002; 236: 397-406.

9. Weber JC, Navarra G, Jiao LR, Nicholls JP, Jensen SL, Habib NA. New technique for liver resection using heat coagulative necrosis. Ann Surg 2002; 36: 560-563.

10. Milicevic M, Bulajic P, Zuvela M, Dervenic C, Basaric D, Galun D. A radiofrequency-assisted minimal blood loss liver parenchyma dissection technique. Dig Surg 2007; 24: 306-313.

11. Stella M, Percivale A, Pasqualini M et al. Radiofrequency-assisted liver resection. J Gastroint Surg 2003; 7: 797-801.

12. Ferko A, Šubrt Z, Havel E, Melicha B, Jon B. Radiofrequency - assisted liver resection. Analysis of a group of consecutive patients treated at a single centre. Rozhl Chir 2007; 5: 228-232.

13. Vávra P, El-Gendi A, Papaevangelou A et al. New approach to the liver metastasis treatment: hand assisted laparoscopic radiofrequency liver resection. Rozhl Chir 2007; 10: 554-557.

14. Parkin DM, Bray F, Ferlay J, Pisani P. Estimating the world cancer burden: GLOBOCAN 2000. Int J Cancer 2001; 94: 153-156.

15. Srivatanakul P, Sriplung H, Deerasamee S. Epidemiology of liver cancer: an overview. Asian Pacific J Cancer Prev 2004; 5: 118-125.

16. Torzilli G, Makuuchi M, Midorikawa Y et al. Liver resection without total vascular exclusion: hazardous or beneficial? An analysis of our experience. Ann Surg 2001; 233: 167-175.

17. Ihnát $P$, Vávra $P$, Dostalík $J$, Guňková $P$, Vávrová $M$, Horáček J. Our experience with radiofrequency assisted liver resection for liver tumors. Rohl Chir 2008; 2: 96-100.

18. Poon RT, Fan ST, Lo CM et al. Improving perioperative outcome expands the role of hepatectomy in management of benign and malignant hepatobiliary diseases. Analysis of 1222 consecutive patients from a prospective database. Ann Surg 2004; 240: 698-710.

19. Belghiti J, Hiramatsu K, Benoist S, Massault PP, Sauvanet A, Farges O. Seven hundred forty seven hepatectomies in the 1990s: an update to evaluate the actual risk of liver resection. J Am Coll Surg 2000; 191: 38-46.

20. Brancatisano R, Isla AM, Habib NA. Is radical hepatic surgery safe? Am J Surg 1998; 175: 161-163.

21. Scatton O, Massault JP, Dousset B et al. Major liver resection without clamping: a prspective reappraisal in the era of modern surgical tools. J Am Coll Surg 2004; 199: 702-708.

22. Weber JC, Bachellier P, Oussoultzglou E, Jaeck D. Simultaneous resection of colorectal primary tumour and synchronous liver metastases. $\mathrm{Br}$ J Surg 2003; 90. 956-962.

23. Imamura $\mathbf{H}$, Seyama $\mathbf{Y}$, Kokudo $\mathbf{N}$ et al. One thousand fifty-six hepatectomies without mortality in 8 years. Arch Surg 2003; 138: 1198-1206.

Received October 12, 2010. Accepted September 20, 2012. 